\title{
Optimal first-line and second-line treatments for metastatic renal cell carcinoma: current evidence
}

This article was published in the following Dove Press journal: International Journal of Nephrology and Renovascular Disease 29 October 2014

Number of times this article has been viewed

Maxine Sun'

Alessandro Larcher ${ }^{1,2}$

Pierre I Karakiewicz'

'Cancer Prognostics and Health Outcomes Unit, University of Montreal Health Center, Montreal, QC, Canada; ${ }^{2}$ Department of Urology, Università Vita-Salute San Raffaele, Milan, Italy
Correspondence: Maxine Sun

Cancer Prognostics and Health Outcomes Unit, 264 Blvd ReneLevesque E Room 228, Montreal, QC, H2X IPI, Canada

Tel +I 5 I48908000 ext 35335

Fax + 5142275103

Email mow.sun@umontreal.ca

\begin{abstract}
Since 2005, an abundance of targeted agents has been approved for the treatment of metastatic renal cell carcinoma ( $\mathrm{mRCC}$ ), without any specification as to what may be the most optimal first-line and second-line sequence. Hence, our objective was to critically examine the evidence supporting the use of first-line and second-line agents in the management of mRCC. Our review suggests that in first line, sunitinib and pazopanib represent treatment options for patients with favorable or intermediate-risk features and clear cell histology. Unfortunately, the Phase III trial cannot conclusively prove the noninferiority of pazopanib relative to sunitinib. Hence, the use of sunitinib as first-line standard of care remains justified. Pazopanib represents an option for specific patients in whom sunitinib might not be tolerated. In patients with poor-risk features, temsirolimus represents the only option supported with level 1 evidence. Less optimal alternatives include sunitinib and bevacizumab combined with interferon, based on the minimal inclusion of poor-risk patients in pivotal Phase III studies of these two molecules. In patients with non-clear cell mRCC, the use of temsirolimus is supported by Phase III data, unlike for any other molecule. In second line, the options consist of everolimus and axitinib. However, the axitinib data are substantially more robust given the inclusion of more patients considered as true second-line, and validly justify the choice of axitinib over everolimus. Nonetheless, the Phase III trial of everolimus may be considered as level 1 evidence for use as third-line or subsequent lines of therapy.
\end{abstract}

Keywords: targeted therapy, metastatic, renal cell carcinoma, clear cell, sequential therapy

\section{Introduction}

The management of metastatic renal cell carcinoma (mRCC) is in constant evolution and mRCC patients have an increasingly greater number of systemic treatment options. Currently, the biggest debate surrounds the selection criteria for first-line and second-line agents in patients with clear cell $\mathrm{mRCC}$, and favorable or intermediate characteristics. Specifically, the greatest controversy focuses on the choice between sunitinib ${ }^{1,2}$ and pazopanib ${ }^{3}$ in first line and use of axitinib ${ }^{4}$ versus everolimus ${ }^{5,6}$ in second line. While evidence level 1 data are available for more than one molecule, existing guidelines indicate several options for first-line and second-line therapy without specifying what may be the ideal first and second choice. $^{7,8}$ The focus of this paper is on objective criteria that could help in the selection of the most optimal first-line or second-line therapy in patients with mRCC.

\section{Selection of first-line treatment}

First-line treatment in patients with favorableto intermediate-risk clear cell mRCC

Currently, Phase III data originating from four randomized trials support the use of three molecules in the first-line setting, ie, sunitinib, ${ }^{1,2}$ pazopanib, ${ }^{3}$ and bevacizumab, 
in combination with interferon- $\alpha .{ }^{9,10}$ The criteria for choosing between sunitinib and pazopanib currently represent the hottest topic of debate. Its importance prompted the design and conduct of the first head-to-head Phase III trial comparing two first-line treatment alternatives for mRCC patients. ${ }^{11}$ Here we review the key findings of this trial. First, we briefly review the key findings of the original pivotal sunitinib and pazopanib trials that prompted the head-to-head comparison.

Sunitinib was originally compared with interferon- $\alpha$ in 750 patients with clear cell $\mathrm{mRCC}$ and predominantly favorable $(n=264 ; 35 \%)$ or intermediate $(n=421 ; 56 \%)$ Memorial Sloan Kettering Cancer Center (MSKCC) risk features randomized to either oral sunitinib at a dose of $50 \mathrm{mg}$ once daily in 6-week cycles consisting of 4 weeks of treatment followed by 2 weeks without treatment, or a subcutaneous injection three times per week on nonconsecutive days at 3 MU per dose during the first week, $6 \mathrm{MU}$ per dose the second week, and $9 \mathrm{MU}$ per dose thereafter. ${ }^{2}$ Of 375 sunitinib-treated patients, $34 \%(\mathrm{n}=127)$ discontinued treatment versus $65 \%$ $(n=234)$ in the interferon- $\alpha$ arm. According to the study's independent central review, no complete response was observed. Partial response and stable disease were recorded in $31 \%(n=103)$ and $48 \%(n=160)$, for an overall response rate of $79 \%(n=263)$ in the sunitinib arm. The median progression-free survival was 11 months versus 5 months, which resulted in a hazard ratio of 0.42 (95\% confidence interval $[\mathrm{CI}] 0.32-0.54, P<0.001$ ) for sunitinib relative to interferon- $\alpha$. In the final updated results of the Phase III sunitinib trial, the investigators showed a median overall survival of 26.4 months versus 21.8 months for sunitinib versus interferon- $\alpha$, respectively (hazard ratio $0.82,95 \%$ CI $0.67-1.00, P=0.051) .{ }^{1}$ In subexploratory analyses, the authors censored 25 patients in the interferon- $\alpha$ group who had crossed over to the sunitinib group during the study, and observed a median overall survival of 26.4 months versus 20.0 months for sunitinib and interferon- $\alpha$, respectively (hazard ratio $0.81,95 \%$ CI $0.66-0.99, P=0.036$ ).

Pazopanib represents the oral alternative to sunitinib for first-line use. Its pivotal data originate from a first-line Phase III trial that randomized 435 predominantly favorable $(\mathrm{n}=170 ; 39 \%)$ or intermediate $(\mathrm{n}=236 ; 54 \%)$ MSKCC risk patients to either pazopanib or placebo. ${ }^{3}$ Of all pazopanibtreated patients, $78 \%(\mathrm{n}=227)$ discontinued treatment versus $90 \%(n=131)$ in the placebo arm. Of the 290 pazopanib-treated patients, only 155 were treatment-naïve $(53 \%)$. The remaining 135 patients received prior cytokines (47\%). To render the results from that trial comparable with those of the sunitinib study, which included only patients who had not received prior treatment with systemic therapy, we focus on the subgroup of patients who were treatment-naïve at enrolment. Partial response and complete response were recorded in $32 \%(n=49)$ of treatment-naïve patients exposed to pazopanib. The median progression-free survival in the treatment-naïve subpopulation was 11.1 months versus 2.8 months for pazopanib and placebo, respectively, corresponding to a hazard ratio of 0.54 (95\% CI 0.35-0.84, $P<0.001$ ).

The relatively small overall sample size treated with pazopanib ( $n=435)$, and most importantly the even smaller proportion of first-line patients who received pazopanib $(n=155)$, as well as the limitations related to indirect trial comparisons, prompted the design of a head-to-head prospective Phase III trial of sunitinib and pazopanib (COMParing the efficacy, sAfety and toleRability of paZopanib vs. sunitinib [COMPARZ], ClinicalTrials.gov identifier NCT00720941). ${ }^{11}$ By focusing on the same characteristics described in the pivotal pazopanib ${ }^{3}$ and sunitinib $b^{1,2}$ trials, the randomized design rested on the noninferiority comparative effectiveness of $800 \mathrm{mg}$ once daily continuous dosing of pazopanib relative to $50 \mathrm{mg}$ once daily $4 / 6$ weeks dosing of sunitinib. For this purpose, a noninferiority margin of 1.25 for assessment of progression-free survival (the primary endpoint) was predefined by the authors. For the ratio not to exceed the 1.25 cut-off, it would imply that a median progression-free survival of 8.8 months with pazopanib and a progression-free survival of 11 months or less with sunitinib would be required. It is noteworthy that the originally planned sample size of 876 patients was meant to provide $80 \%$ power to confirm noninferiority of pazopanib. However, this protocol was quickly amended to increase the sample to 1,100 patients in order to maintain a power of $80 \%$, with a target event count of 631 required disease progressions. Instead of reopening enrolment, the authors supplemented the original cohort with an additional cohort of 160 Asian patients enrolled in an ongoing Phase II study with identical trial design and patient selection criteria (ClinicalTrials.gov identifier NCT01147822).

The primary intent-to-treat analyses based on 557 pazopanib patients and 553 sunitinib patients showed a median progression-free survival of 9.5 months $(95 \% \mathrm{CI}$ 8.3-11.1) for sunitinib and 8.4 months (95\% CI 8.3-10.9) for pazopanib, corresponding to a hazard ratio of 1.047 with an upper limit CI level of 1.22. Since the latter did not exceed the noninferiority threshold value of 1.25 , pazopanib was considered statistically noninferior to sunitinib. It is worth mentioning that the per-protocol analysis based on 
501 pazopanib patients and 494 sunitinib patients showed a median progression-free survival of 10.2 months for sunitinib versus 8.4 months for pazopanib. The corresponding hazard ratio was 1.069 with an upper limit CI level of 1.255 , which actually exceeded the noninferiority margin of 1.25 . Within this independent review study assessment, three and one complete responses were observed for sunitinib and pazopanib, respectively. Partial response and stable disease in the intent-to-treat population were recorded in $24 \%$ and $44 \%$ of sunitinib patients versus $39 \%$ and $31 \%$ of pazopanib patients, resulting in an overall response rate of $69 \%$ versus $71 \%$ for sunitinib and pazopanib, respectively. Overall, 502 deaths occurred. Median overall survival at the time of the trial was 28.4 months versus 29.3 months for pazopanib and sunitinib, respectively (hazard ratio $0.91,95 \% \mathrm{CI} 0.76-1.08, P=0.28$ ), thus showing a very small nonsignificant difference. The proportion of patients who discontinued intervention due to adverse events was $24 \%$ versus $20 \%$ for pazopanib versus sunitinib, respectively. The higher rate of discontinuation observed for pazopanib relative to sunitinib was mainly due to liver-related events (6\% versus $1 \%$ ). However, overall, pazopanib patients less frequently reported most of the examined toxicity types. Health-related quality of life data showed less severe changes in baseline values for pazopanib relative to sunitinib. The authors concluded that pazopanib was noninferior to sunitinib with respect to progression-free survival and that safety and quality of life profiles were in favor of pazopanib.

Although there is no doubt that direct comparison of sunitinib versus pazopanib represents a highly laudable effort aimed at providing evidence level 1 data in regards to two management alternatives for clear cell mRCC patients, many questions pertaining to study results and their interpretation remain unanswered. First, it is worrisome that the results of the per-protocol analysis differ from those of the intent-to-treat analysis, where pazopanib failed to confirm noninferiority in that subanalysis, as the upper limit of the CI exceeded the predefined 1.25 threshold. The discrepancy between the perprotocol and intent-to-treat analyses is noteworthy, as noninferiority trials emphasize the importance of results obtained in the per-protocol analysis and their consistency with the results obtained in the intent-to-treat primary analyses. ${ }^{12}$ Second, the predefined threshold for declaring pazopanib statistically noninferior to sunitinib was considerable $(25 \%)$. It is questionable whether, in real clinical practice, patients would be willing to accept a novel treatment modality that may translate into a $22 \%$ (intent-to-treat analysis) or even a $25 \%$ (per-protocol analysis) higher rate of progression than sunitinib. Third, the results of the noninferiority trial also need to be interpreted in light of the timing of disease progression (every 6 weeks until week 24 , and every 12 weeks thereafter until progression) and of health-related quality of life (at 4 weeks of 6 week cycle) assessments. These time points are clearly the worst times for assessing efficacy or health-related quality of life on sunitinib. Primarily, regarding disease progression, the offtreatment period between weeks 4 and 6 is associated with a $10 \%$ progression rate. ${ }^{13}$ Consequently, efficacy measurements at 6 weeks reflect unfavorably on sunitinib. In this context, the effect of sunitinib on health-related quality of life is at its worst at 4 weeks of each cycle, exactly the time when healthrelated quality of life is measured. These considerations do not apply to pazopanib, given that its dosing is continuous whereas the dosing of sunitinib is intermittent. Such non-negligible differences are important to take into consideration during study design. In addition to such potential pitfalls, it remains to be that the upper bound of the CI reflecting the efficacy of pazopanib (intent-to-treat 1.22) was close to being outside the range of what is considered noninferior to sunitinib (intent-totreat 1.22), or even at the limit (per-protocol 1.255). Hence, it may be stated that pazopanib might not be conclusively considered as noninferior to sunitinib. ${ }^{12}$

As a consequence of the COMPARZ data, which failed to conclusively demonstrate noninferiority of pazopanib relative to sunitinib, the choice to opt for pazopanib instead of sunitinib as the first-line option cannot be justified. It is important to highlight that pazopanib should remain an option for patients who are particularly predisposed to some of the toxicities of sunitinib, such as hematological toxicities (anemia, thrombocytopenia, or neutropenia), hypothyroidism, or hand-foot syndrome, since these toxicities were recorded significantly more frequently with sunitinib in the Phase III trial. ${ }^{11}$ Moreover, some patients might prefer pazopanib over sunitinib. ${ }^{14}$

It should also be noted that despite the emphasis on orally administered tyrosine kinase inhibitor (TKI) agents (eg, sunitinib and pazopanib), bevacizumab combined with interferon- $\alpha^{9,10,15,16}$ also represents an effective first-line alternative. Nonetheless, the need for intravenous administration represents one of its major drawbacks. Moreover, the lack of comparative effectiveness data against sunitinib with respect to efficacy and tolerability, especially in light of available data comparing sunitinib versus pazopanib, weakens the rationale to consider bevacizumab with interferon- $\alpha$ as a first-line option.

In conclusion, three molecules (sunitinib, pazopanib, and bevacizumab combined with interferon- $\alpha$ ) designed for 
patients with clear cell $\mathrm{mRCC}$ and favorable to intermediate MSKCC risk were tested in Phase III trials. ${ }^{1-3,9,10,15,16}$ Of these, sunitinib and pazopanib were examined in a head-to-head noninferiority trial. ${ }^{11}$ This trial could not conclusively confirm the noninferiority of pazopanib relative to sunitinib. Nonetheless, it allowed a prospective assessment of these alternative systemic strategies. Such direct comparisons are not available for bevacizumab.

\section{First-line treatment in patients with poor-risk or non-clear cell mRCC}

Patients with poor-risk features according to the MSKCC risk profiles developed by Motzer et al during the cytokine era ${ }^{17}$ (at least of the following six predictors: serum lactate dehydrogenase more than 1.5 times the upper limit of the normal range; a hemoglobin level below the lower limit of the normal range; a corrected serum calcium level $>10 \mathrm{mg} / \mathrm{dL}$ [2.5 mmol/L]; a time from initial diagnosis of $\mathrm{mRCC}$ to randomization of less than one year; a Karnofsky performance score of 60 or 70 ; metastases in multiple organs), or the more contemporary risk profile developed by Heng et $\mathrm{al}^{18}$ in the targeted therapy era (three to six of the following predictors: hemoglobin less than the lower limit of normal; corrected calcium greater than the upper limit of normal; Karnofsky performance score less than $80 \%$; time from diagnosis to treatment of less than one year; neutrophils greater than the upper limit of normal; platelets greater than the upper limit of normal), have been examined in the randomized Phase III study $(n=626)$ investigating the efficacy of temsirolimus alone $(n=209)$ versus temsirolimus with interferon $(n=210)$ versus interferon alone $(n=207) .{ }^{19}$ The study also included 124 patients with non-clear cell histology, which makes it the only randomized trial examining treatment efficacy and tolerability in this particular patient subgroup.

Median overall survival was 10.9 months for temsirolimus alone versus 8.4 months for temsirolimus in combination with interferon versus 7.3 months for interferon alone. The objective response rates were $8.6 \%$ versus $8.1 \%$ versus $4.8 \%$ for temsirolimus alone, temsirolimus with interferon, and interferon alone, respectively (not significantly different). The calculated objective response and stable disease rates for $\geq 24$ weeks were $32 \%, 28 \%$, and $16 \%$ for temsirolimus alone, temsirolimus with interferon, and interferon alone, respectively. The median progression-free survival was 3.8 months for temsirolimus alone versus 3.7 months and 1.9 months for temsirolimus with interferon and interferon alone, respectively.

Grade 3 or 4 toxicity was significantly lower in those treated with temsirolimus alone (67\%), compared with $78 \%$ for patients in the interferon alone group and $87 \%$ for patients in the temsirolimus combined with interferon group (both $P=0.02)$. In exploratory subgroup analyses, an interaction between treatment and age, baseline serum lactate dehydrogenase was observed. Specifically, the effect of temsirolimus on overall survival was greater among patients aged younger than 65 years, in those with a serum lactate dehydrogenase level of more than 1.5 times the upper limit of normal, and in those with non-clear cell mRCC. ${ }^{19}$

In an important exploratory subgroup analysis from the Phase III trial of temsirolimus, ${ }^{19}$ Dutcher et $\mathrm{al}^{20}$ compared temsirolimus with interferon in 73 patients with non-clear cell mRCC. The median overall survival was 11.6 months versus 4.3 months for temsirolimus compared with interferon, respectively (hazard ratio 0.49 , 95\% CI 0.29-0.85). The median progression-free survival was 7.0 months versus 1.8 months for the same groups, respectively (hazard ratio $0.38,95 \%$ CI $0.23-0.62$ ). These findings represent the cornerstone of the rationale for use of temsirolimus in patients with non-clear cell $\mathrm{mRCC}$, given that no other randomized trial has addressed this patient category. In consequence, temsirolimus not only represents the standard first-line agent of choice for patients with poor-risk clear cell $\mathrm{mRCC}$, but its Phase III-based recommendation also extends to those with non-clear cell mRCC variants.

What are the alternatives, when temsirolimus cannot be administered to poor-risk patients? It is of note that 48 poorrisk patients were included in the pivotal Phase III trial of sunitinib. ${ }^{2}$ Similarly, poor-risk patients were also included in both the Phase III trials of bevacizumab combined with interferon, ie, 52 in the Avastin and Roferon in Renal Cell Carcinoma (AVOREN) trial (ClinicalTrials.gov identifier NCT02056587 $)^{9,15}$ and 75 in the Cancer and Leukemia Group B (CALGB) trial (ClinicalTrials.gov identifier NCT00072046). ${ }^{10,16}$ In consequence, the use of sunitinib or bevacizumab combined with interferon in patients with poorrisk $\mathrm{mRCC}$ can be justified if temsirolimus cannot be used. Conversely, only 14 patients with poor-risk features were included in the Phase III trial of pazopanib versus placebo. ${ }^{3}$ Therefore, the consideration of pazopanib for patients with poor-risk characteristics is supported by very limited evidence, and its use might be difficult to recommend over secondary options, such as sunitinib or bevacizumab with interferon.

Taken together, temsirolimus represents the standard of care in patients with poor-risk characteristics and/or nonclear cell histological subtype (level 1 evidence). Alternatives include sunitinib, bevacizumab combined with interferon, and pazopanib, if temsirolimus is not available. 


\section{Selection of second-line treatment}

Treatment sequencing in second-line after first-line TKI failure may consist of either a second-line TKI or a secondline mammalian target of rapamycin (mTOR) inhibitor. The following sections review the existing data supporting either sequencing approach.

\section{Use of second-line TKI after first-line TKI failure}

The rationale for sequential use of two TKI originates from numerous case series that examined the efficacy of sunitinib followed by sorafenib. ${ }^{21-24}$ In those series, median progression-free survival on first-line sunitinib ranged from 5.1 to 8.6 months. The median progression-free survival on secondline sorafenib ranged from 2.9 to 8.9 months. A prominent study is Investigating Torisel As Second-Line Therapy (INTORSECT) (ClinicalTrials.gov identifier NCT00474786), a randomized Phase III trial comparing temsirolimus versus sorafenib as a second-line treatment option in patients with $\mathrm{mRCC}$ who had progressed on sunitinib in the first-line setting. ${ }^{25}$ In that report, the authors noted a significant overall survival difference in favor of sorafenib compared with temsirolimus (hazard ratio $1.31,95 \%$ CI $1.05-1.63, P=0.01$ ). The longer overall survival duration observed with sorafenib suggests that sequencing with vascular endothelial growth factor receptor (a TKI) may be more optimal than sequencing with a mammalian target TKI.

Phase III data supporting the use of a TKI after previous TKI failure have been examined in the AXIS (ClinicalTrials. gov identifier NCT00678392) study. ${ }^{4}$ The latter comprised 723 patients with clear cell $\mathrm{mRCC}$ who previously failed sunitinib, temsirolimus, cytokines, or bevacizumab with interferon. Second-line TKI therapy consisted of either axitinib $5 \mathrm{mg}$ twice daily or sorafenib $400 \mathrm{mg}$ twice daily. No complete response was reported. Partial response and stable disease for $\geq 20$ weeks was $19 \%$ and $27 \%$ for axitinib versus $9 \%$ and $21 \%$ for sorafenib, respectively, corresponding to an objective response rate of $19 \%$ versus $9 \%$. The median progression-free survival was 6.7 months versus 4.7 months for axitinib and sorafenib, respectively (hazard ratio 0.67 , $95 \% \mathrm{CI} 0.54-0.81, P<0.001)$. In analyses stratified according to the previous treatment regimen, 194 patients were sunitinibrefractory $(54 \%)$ in the axitinib arm $(n=361)$ compared with 195 patients (54\%) in the sorafenib arm $(n=362)$. The results showed that the median progression-free survival among those who failed a previous sunitinib regimen was 4.8 months versus 3.4 months for axitinib versus sorafenib, respectively (hazard ratio $0.74,95 \% \mathrm{CI} 0.57-0.96, P=0.01$ ).
Overall, axitinib and sorafenib were well tolerated. The most common nonhematological grade $\geq 3$ toxicities for axitinib and sorafenib were hypertension ( $16 \%$ versus $11 \%)$, fatigue $(11 \%$ versus $5 \%)$, and diarrhea (11\% versus $7 \%)$. Overall, 22 patients discontinued treatment due to an adverse event in the axitinib arm compared with 33 in the sorafenib arm. The most common reasons for discontinuation were dyspnea, nausea, fatigue, hypertension, and vomiting. There were no treatment-related deaths.

The AXIS study represents the first head-to-head, second-line comparison of a novel molecule against an already established second-line agent with proven efficacy, ie, sorafenib. ${ }^{26,27}$ In this study, axitinib demonstrated a better median progression-free survival when compared with sorafenib. Moreover, the majority (54\%) of patients exposed to either axitinib or sorafenib received first-line sunitinib therapy, which is consistent with contemporary practice guidelines that support first-line use of sunitinib. ${ }^{8}$ In consequence, the AXIS study currently provides the most robust and definitive data for second-line use following first-line failure with sunitinib.

\section{Use of second-line mTOR inhibitor after TKI failure}

The Renal Cell cancer treatment with Oral RAD001 given Daily (RECORD-1) (ClinicalTrials.gov identifier NCT00410124) study provided data regarding sequential use of everolimus in 272 patients ( $10 \mathrm{mg}$ once daily) versus 138 patients on placebo, in conjunction with best supportive care., ${ }^{5,6}$ Partial response and stable disease rates were captured in three and 171 of the everolimus patients, respectively. Median progression-free survival was 4.9 months for everolimus versus 1.9 months for placebo (hazard ratio $0.33,95 \%$ CI $0.25-0.43$ ). The median overall survival was 14.8 months for everolimus versus 14.4 months for placebo, where $80 \%$ of patients in the placebo arm crossed over to the everolimus group.

However, it should be noted that most of the included patients who received everolimus were not truly second-line. Specifically, 82 patients received everolimus in fifth line, 104 patients in fourth line, and 141 patients in third line., ${ }^{5,28}$ Only $21 \%(n=89)$ of patients received everolimus in the second-line setting. Furthermore, second-line everolimus after failure of sunitinib was only administered to 43 patients, with a median progression-free survival of 4.6 months versus 1.8 months in 13 patients treated with placebo after failure of sunitinib (hazard ratio $0.22,95 \% \mathrm{CI} 0.09-0.55$ ).

The results originating from the RECORD-1 study have provided level 1 evidence for efficacy of sequential targeted therapies, but predominantly in the third and subsequent lines. 
Indeed, true second-line everolimus data were based on a small number of patients $(n=89)$, therefore providing only partial proof regarding the specific efficacy of this molecule in the second-line setting. The number of patients was reduced even further when considering true second-line everolimus after failure of sunitinib $(n=43)$ or pazopanib $(n=0)$, ie, the primary first-line options in clear cell $\mathrm{mRCC}$. In consequence, based on the RECORD-1 study data, everolimus represents the molecule of choice for third-line use or in subsequent lines, since $79 \%$ of the included patients had failed two previous lines of therapy prior to receiving everolimus.

Finally, a concerning bias of the RECORD-1 study is that one of the study inclusion criteria specifies that only patients who had progressed on or within 6 months of stopping treatment with sunitinib or sorafenib, or both drugs, may be considered eligible. ${ }^{6}$ Hence, the analyzed group likely comprised TKI nonresponders. This makes consideration of everolimus as first-choice second-line difficult given that the majority of patients would be given either sunitinib or pazopanib (both TKIs) in the first-line setting.

Taken together, two Phase III trials tested the efficacy of sequential therapy in patients with mRCC. Although both everolimus and axitinib demonstrated improved progressionfree survival compared with their respective placebo and sorafenib counterparts, the difference lies in the sequence of administration. In comparison with the AXIS study, the evidence originating from the RECORD-1 study for administration of everolimus in the second-line setting after TKI failure may be limited. In contrast, more true second-line patients were included in the axitinib trial. That said, it is important to note that currently neither everolimus nor axitinib have demonstrated prolonged overall survival compared with either placebo (even after censoring patients who crossed over) or sorafenib (data not yet mature).

\section{Conclusion}

The objective of this paper was to critically examine the evidence supporting the use of first-line and secondline agents in the management of mRCC. In first-line, sunitinib and pazopanib represent treatment options for patients with favorable or intermediate-risk features and clear cell histology. Phase III data cannot conclusively demonstrate the noninferiority of pazopanib relative to sunitinib. Hence, use of sunitinib as first-line standard of care remains justified. Pazopanib represents an option for specific patients in whom sunitinib might not be tolerated. In patients with poor-risk features, temsirolimus is the only option supported with level 1 evidence. Less optimal alternatives include sunitinib and bevacizumab combined with interferon, based on the minimal inclusion of poorrisk patients in the pivotal Phase III studies of these two molecules. In patients with non-clear cell mRCC, use of temsirolimus is supported by Phase III data, unlike for any other molecule. Second-line options consist of everolimus and axitinib. However, the axitinib data are substantially more robust given the inclusion of more patients considered as genuinely second-line, and justify the choice of axitinib over everolimus. Nonetheless, the Phase III trial of everolimus may be considered as level 1 evidence for use in third or subsequent lines of therapy.

\section{Disclosure}

Dr Karakiewicz has received honoraria from Pfizer and GlaxoSmithKline. The authors report no other conflicts of interest in this work.

\section{References}

1. Motzer RJ, Hutson TE, Tomczak P, et al. Overall survival and updated results for sunitinib compared with interferon alfa in patients with metastatic renal cell carcinoma. J Clin Oncol. 2009;27(22): 3584-3590.

2. Motzer RJ, Hutson TE, Tomczak P, et al. Sunitinib versus interferon alfa in metastatic renal-cell carcinoma. $N$ Engl J Med. 2007;356(2): $115-124$.

3. Sternberg CN, Davis ID, Mardiak J, et al. Pazopanib in locally advanced or metastatic renal cell carcinoma: results of a randomized Phase III trial. J Clin Oncol. 2010;28(6):1061-1068.

4. Rini BI, Escudier B, Tomczak P, et al. Comparative effectiveness of axitinib versus sorafenib in advanced renal cell carcinoma (AXIS): a randomised Phase 3 trial. Lancet. 2011;378(9807):1931-1939.

5. Motzer RJ, Escudier B, Oudard S, et al. Phase 3 trial of everolimus for metastatic renal cell carcinoma: final results and analysis of prognostic factors. Cancer. 2010;116(18):4256-4265.

6. Motzer RJ, Escudier B, Oudard S, et al. Efficacy of everolimus in advanced renal cell carcinoma: a double-blind, randomised, placebocontrolled Phase III trial. Lancet. 2008;372(9637):449-456.

7. National Comprehensive Cancer Network, Kidney Cancer, version 2.2014. Available from: http://www.jnccn.org/content/12/2/175. full. Accessed July 1, 2014.

8. Escudier B, Eisen T, Porta C, et al. Renal cell carcinoma: ESMO clinical practice guidelines for diagnosis, treatment and follow-up. Ann Oncol. 2012;23 Suppl 7:vii65-vii71.

9. Escudier B, Bellmunt J, Negrier S, et al. Phase III trial of bevacizumab plus interferon alfa-2a in patients with metastatic renal cell carcinoma (AVOREN): final analysis of overall survival. J Clin Oncol. 2010;28(13): 2144-2150.

10. Rini BI, Halabi S, Rosenberg JE, et al. Phase III trial of bevacizumab plus interferon alfa versus interferon alfa monotherapy in patients with metastatic renal cell carcinoma: final results of CALGB 90206. J Clin Oncol. 2010;28(13):2137-2143.

11. Motzer RJ, McCann L, Deen K. Pazopanib versus sunitinib in renal cancer. N Engl J Med. 2013;369(20):1970.

12. Piaggio G, Elbourne DR, Pocock SJ, et al. Reporting of noninferiority and equivalence randomized trials: extension of the CONSORT 2010 statement. JAMA. 2012;308(24):2594-2604.

13. Wolter P, Beuselinck B, Pans S, et al. Flare-up: an often unreported phenomenon nevertheless familiar to oncologists prescribing tyrosine kinase inhibitors. Acta Oncol. 2009;48(4):621-624. 
14. Escudier B, Porta C, Bono P, et al. Randomized, controlled, double-blind, cross-over trial assessing treatment preference for pazopanib versus sunitinib in patients with metastatic renal cell carcinoma: PISCES study. J Clin Oncol. April 31, 2014. [Epub ahead of print.]

15. Escudier B, Pluzanska A, Koralewski P, et al. Bevacizumab plus interferon alfa-2a for treatment of metastatic renal cell carcinoma: a randomised, double-blind Phase III trial. Lancet. 2007;370(9605): 2103-2111.

16. Rini BI, Halabi S, Rosenberg JE, et al. Bevacizumab plus interferon alfa compared with interferon alfa monotherapy in patients with metastatic renal cell carcinoma: CALGB 90206. J Clin Oncol. 2008;26(33): 5422-5428.

17. Motzer RJ, Bacik J, Murphy BA, et al. Interferon-alfa as a comparative treatment for clinical trials of new therapies against advanced renal cell carcinoma. J Clin Oncol. 2002;20(1):289-296.

18. Heng DY, Xie W, Regan MM, et al. Prognostic factors for overall survival in patients with metastatic renal cell carcinoma treated with vascular endothelial growth factor-targeted agents: results from a large, multicenter study. J Clin Oncol. 2009;27(34):5794-5799.

19. Hudes G, Carducci M, Tomczak P, et al. Temsirolimus, interferon alfa, or both for advanced renal-cell carcinoma. $N \mathrm{Engl} \mathrm{J} \mathrm{Med}$. 2007;356(22):2271-2281.

20. Dutcher JP, Souza P, McDermott D, et al. Effect of temsirolimus versus interferon- $\alpha$ on outcome of patients with advanced renal cell carcinoma of different tumor histologies. Med Oncol. 2009;26(2):202-209.
21. Tamaskar I, Garcia JA, Elson P, et al. Antitumor effects of sunitinib or sorafenib in patients with metastatic renal cell carcinoma who received prior antiangiogenic therapy. J Urol. 2008;179(1):81-88.

22. Sablin MP, Negrier S, Ravaud A, et al. Sequential sorafenib and sunitinib for renal cell carcinoma. J Urol. 2009;182(1):29-34.

23. Dudek AZ, Zolnierek J, Dham A, et al. Sequential therapy with sorafenib and sunitinib in renal cell carcinoma. Cancer. 2009;115(1): 61-67.

24. Porta C, Procopio G, Carteni G, et al. Sequential use of sorafenib and sunitinib in advanced renal-cell carcinoma (RCC): an Italian multicentre retrospective analysis of 189 patient cases. BJU Int. 2011;108(8 Pt 2): E250-E257.

25. Hutson TE, Escudier B, Esteban E, et al. Randomized Phase III trial of temsirolimus versus sorafenib as second-line therapy after sunitinib in patients with metastatic renal cell carcinoma. J Clin Oncol. 2014;32(8): 760-767.

26. Escudier B, Eisen T, Stadler WM, et al. Sorafenib in advanced clear-cell renal-cell carcinoma. $N$ Engl J Med. 2007;356(2):125-134.

27. Escudier B, Eisen T, Stadler WM, et al. Sorafenib for treatment of renal cell carcinoma: final efficacy and safety results of the Phase III treatment approaches in renal cancer global evaluation trial. J Clin Oncol. 2009;27(20):3312-3318.

28. Zustovich F, Lombardi G, Nicoletto O, et al. Second-line therapy for refractory renal-cell carcinoma. Crit Rev Oncol Hematol. 2012;83(1) $112-122$.

\section{Publish your work in this journal}

The International Journal of Nephrology and Renovascular Disease is an international, peer-reviewed open-access journal focusing on the pathophysiology of the kidney and vascular supply. Epidemiology, screening, diagnosis, and treatment interventions are covered as well as basic science, biochemical and immunological studies. The journal welcomes original research, clinical studies, reviews \& evaluations, expert opinion and commentary, case reports and extended reports. The manuscript management system is completely online and includes a very quick and fair peerreview system, which is all easy to use. Visit http://www.dovepress.com/ testimonials.php to read real quotes from published authors 